\title{
Electron and hole transport in the organic small molecule a-NPD
}

Cite as: Appl. Phys. Lett. 110, 073301 (2017); https://doi.org/10.1063/1.4976205

Submitted: 28 November 2016 . Accepted: 31 January 2017 . Published Online: 13 February 2017

R. Rohloff, N. B. Kotadiya, N. I. Crăciun, P. W. M. Blom (D, and G. A. H. Wetzelaer
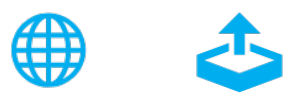

\section{ARTICLES YOU MAY BE INTERESTED IN}

Organic electroluminescent diodes

Applied Physics Letters 51, 913 (1987); https://doi.org/10.1063/1.98799

Hole transport in the organic small molecule material $\alpha$-NPD: evidence for the presence of correlated disorder

Journal of Applied Physics 107, 113710 (2010); https://doi.org/10.1063/1.3407561

Ab initio modeling of steady-state and time-dependent charge transport in hole-only a-NPD devices

Applied Physics Letters 109, 243301 (2016); https://doi.org/10.1063/1.4971969

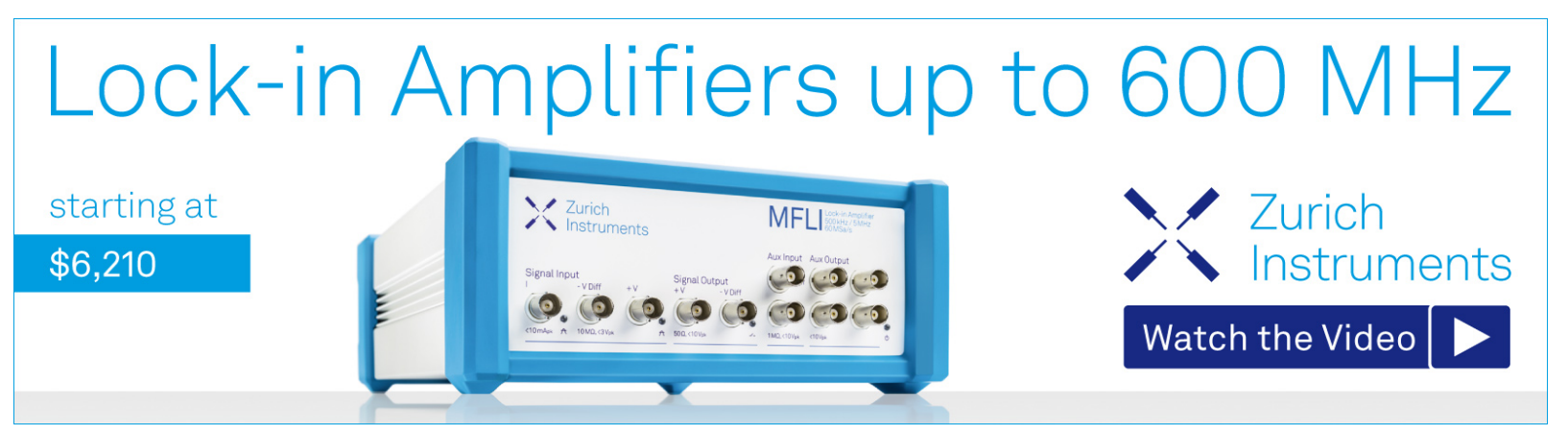




\title{
Electron and hole transport in the organic small molecule $\alpha$-NPD
}

\author{
R. Rohloff, N. B. Kotadiya, N. I. Crăciun, P. W. M. Blom, and G. A. H. Wetzelaer \\ Max Planck Institute for Polymer Research, Ackermannweg 10, 55128 Mainz, Germany
}

(Received 28 November 2016; accepted 31 January 2017; published online 13 February 2017)

\begin{abstract}
Electron and hole transport properties of the organic small molecule $\mathrm{N}, \mathrm{N}^{\prime}$-Di(1-naphthyl)-N, $\mathrm{N}^{\prime}$ diphenyl-(1,1'-biphenyl)-4,4'-diamine are investigated by space-charge-limited current measurements. The hole transport shows trap-free behavior with a mobility of $2.3 \times 10^{-8} \mathrm{~m}^{2} / \mathrm{Vs}$ at vanishing carrier density and electric field. The electron transport, on the other hand, shows heavily trap-limited behavior, which leads to highly unbalanced transport. A trap concentration of $1.3 \times 10^{24} \mathrm{~m}^{-3}$ was found by modeling the electron currents, similar to the universal trap concentration found in conjugated polymers. This indicates that electron trapping is a generic property of organic semiconductors, ranging from vacuum-deposited small-molecules to solution-processed conjugated polymers. Published by AIP Publishing. [http://dx.doi.org/10.1063/1.4976205]
\end{abstract}

Since the fabrication of the first light-emitting diodes (OLEDs) and solar cells based on organic semiconductors, ${ }^{1,2}$ extensive efforts have been made to understand the physical properties of these materials. One of the most important properties of organic semiconductors is the transport of charge carriers.

Charge transport in organic semiconductors is usually characterized by hopping transport between states that have a certain distribution in energy. This energetic disorder stems from the morphological ordering of the molecules and is frequently assumed to have a Gaussian distribution. ${ }^{3}$ Current theoretical research pursues to predict the energetic disorder and mobility by starting from microscopic simulations of the morphology, paving the way to predictive modeling of organic devices. ${ }^{4-6}$ To validate the theoretical predictions, a systematic series of experimental charge transport data is required, including the temperature, electric-field, and layer-thickness dependence of the charge-carrier mobility. Moreover, charge transport can be greatly influenced by charge trapping, caused by the presence of extrinsic impurities, which may result in large deviations between the theoretical predictions and experimental data.

In solution-processed conjugated polymers, the hole transport usually shows trap-free behavior, while the electron transport is severely hindered by charge trapping, which additionally gives rise to nonradiative-recombination losses in polymer-based devices. ${ }^{7,8}$ The electron trapping appears to be caused by extrinsic defects, situated at an energy of around $3.6 \mathrm{eV}$ below the vacuum level. The chemical origin of these extrinsic defects is still not fully understood.

To date, it is unclear if such unbalanced transport and trapping behavior also exists in evaporated organic small molecules, as they are widely used in OLED stacks. Characterization of the hole transport in small molecule organic semiconductors is typically carried out using time-of-flight measurements. ${ }^{9}$ However, for this technique, film thicknesses of several micrometers are required, much thicker than the layers in actual OLED devices. Moreover, charge transport in disordered organic semiconductors frequently shows dispersive behavior, which can lead to an overestimation of the mobility in transient measurements. ${ }^{10}$ In addition, the dependence of the mobility on charge concentration cannot be addressed with the time-of-flight technique, which plays a large role in thin films. ${ }^{11}$ Furthermore, it has been observed that time-of-flight measurements are insensitive to deep traps, ${ }^{12}$ which can severely hinder charge transport. ${ }^{7}$

Therefore, steady-state current-voltage measurements on thin-film single-carrier devices could provide a more relevant characterization of the charge transport. In OLEDs, the current is directly determined by the individual single-carrier mobilities and trapping behavior. In the literature, there have been limited attempts to model the charge transport in organic small molecules in single-carrier devices. Hole-only devices of $\mathrm{N}, \mathrm{N}^{\prime}$-Di(1-naphthyl)-N, $\mathrm{N}^{\prime}$-diphenyl-(1,1'-biphenyl)-4, $4^{\prime}$-diamine $(\alpha$-NPD) have been characterized by using different hopping-transport models. ${ }^{13}$ However, these particular devices exhibited injection barriers, which have a large effect on the measured current density. The presence of an injection barrier therefore complicates a direct characterization of charge transport. ${ }^{5}$ Electron transport has previously been characterized in electron-only devices based on the aluminum complexes $\mathrm{Alq}_{3}$ and BAlq, which showed that electron trapping plays a role in the transport in these molecules. ${ }^{14,15}$ However, it is not clear if electron trapping is specific for Alq-based materials or a general phenomenon occurring in evaporated small molecules, as is the case for solution-processed conjugated polymers.

Here, we investigate the hole and electron transport in single-carrier devices based on the archetypical amorphous ${ }^{16,17}$ hole-transport molecule $\alpha$-NPD. By ensuring barrierless hole injection, we observe trap-free space-charge-limited hole currents, which are modeled with the extended Gaussian disorder model (EDGM). The steady-state mobility is found to be in close agreement with reported time-of-flight mobilities. The electron transport, on the other hand, is observed to be severely hindered by charge trapping, leading to highly unbalanced charge transport.

For a single-carrier device with an ohmic contact, the current is limited by space charge. The current density is given by ${ }^{18}$ 


$$
J=\frac{9}{8} \varepsilon \mu \frac{V^{2}}{L^{3}}
$$

with $J$ the current density, $\varepsilon$ the permittivity, $\mu$ the chargecarrier mobility, $V$ the voltage, and $L$ the layer thickness. As can be seen from Eq. (1), the current scales with thickness to the third power. Therefore, to fulfill the condition of a spacecharge-limited current, it is essential that the layer-thickness dependence is satisfied. In previous work, it was concluded that the hole mobility in $\alpha$-NPD increases with layer thickness. ${ }^{19,20}$ However, this could well be an indication of a barrier at the hole-injection contact: with increasing layer thickness, the bulk resistance increases, reducing the effect of an injection barrier, which leads to an apparent thickness dependence of the mobility when analyzing the current with Eq. (1).

To investigate the hole transport, hole-only devices were prepared, in which the $\alpha$-NPD (sublimed grade, SigmaAldrich) layer is sandwiched between a spin-coated poly(3,4ethylenedioxythiophene):polystyrene sulfonate (PEDOT:PSS) bottom contact and a $\mathrm{MoO}_{3} / \mathrm{Al}$ top contact. After deposition of PEDOT:PSS, the devices were kept in a nitrogen atmosphere and were not exposed to air. All subsequent layers were thermally evaporated in a vacuum at base pressures lower than $1 \times 10^{-6}$ mbar. Electrical characterization of the devices was performed in a controlled $\mathrm{N}_{2}$ atmosphere with a Keithley 2400 source meter. From a work function point of view, the hole current injected from PEDOT:PSS $(5.1 \mathrm{eV})$ into the HOMO of $\alpha$-NPD $(-5.4 \mathrm{eV})$ is expected to be injection limited, whereas $\mathrm{MoO}_{3}$, having a work function of $6.86 \mathrm{eV},{ }^{21}$ should provide an ohmic contact. Remarkably, as displayed in Fig. 1, the hole current injected from $\mathrm{MoO}_{3}$ in forward bias was observed to be lower than the current injected from PEDOT:PSS in reverse bias, for which the reason is unclear.

In order to improve the injection from the $\mathrm{MoO}_{3} / \mathrm{Al}$ top electrode, a $5 \mathrm{~nm}$ layer of tris(4-carbazoyl-9-ylphenyl)amine



FIG. 1. Experimental current density-voltage characteristics of hole-only devices with an $\alpha$-NPD layer thickness of $100 \mathrm{~nm}$, with (circles) and without (squares) a $5 \mathrm{~nm}$ TCTA hole-injection layer. The bottom electrode is grounded, so that holes are injected from $\mathrm{MoO}_{3}$ in forward bias and from PEDOT:PSS in reverse bias. The inset shows a schematic energy-band diagram of the device.
(TCTA) is inserted between $\alpha$-NPD and $\mathrm{MoO}_{3}$. The addition of a thin TCTA layer results in improved hole injection into $\alpha$-NPD, as evidenced by the improved current in forward bias, which now has a quadratic dependence on voltage. To verify if the observed currents are truly space-charge limited, the thickness and temperature dependence were investigated, as depicted in Fig. 2.

In disordered organic semiconductors, the chargecarrier mobility is dependent on the carrier density and the electric field. For hopping transport in a system with localized states that have a Gaussian distribution in energy, the density, field, and temperature dependence of the mobility is described by the extended Gaussian disorder model (EDGM). ${ }^{22}$ The parameters that describe transport in the EDGM are the width of the density-of-states distribution $\sigma$, the lattice constant $a$, and a mobility prefactor $\mu_{\infty}$. The temperature-dependent mobility at zero field and density is then given by
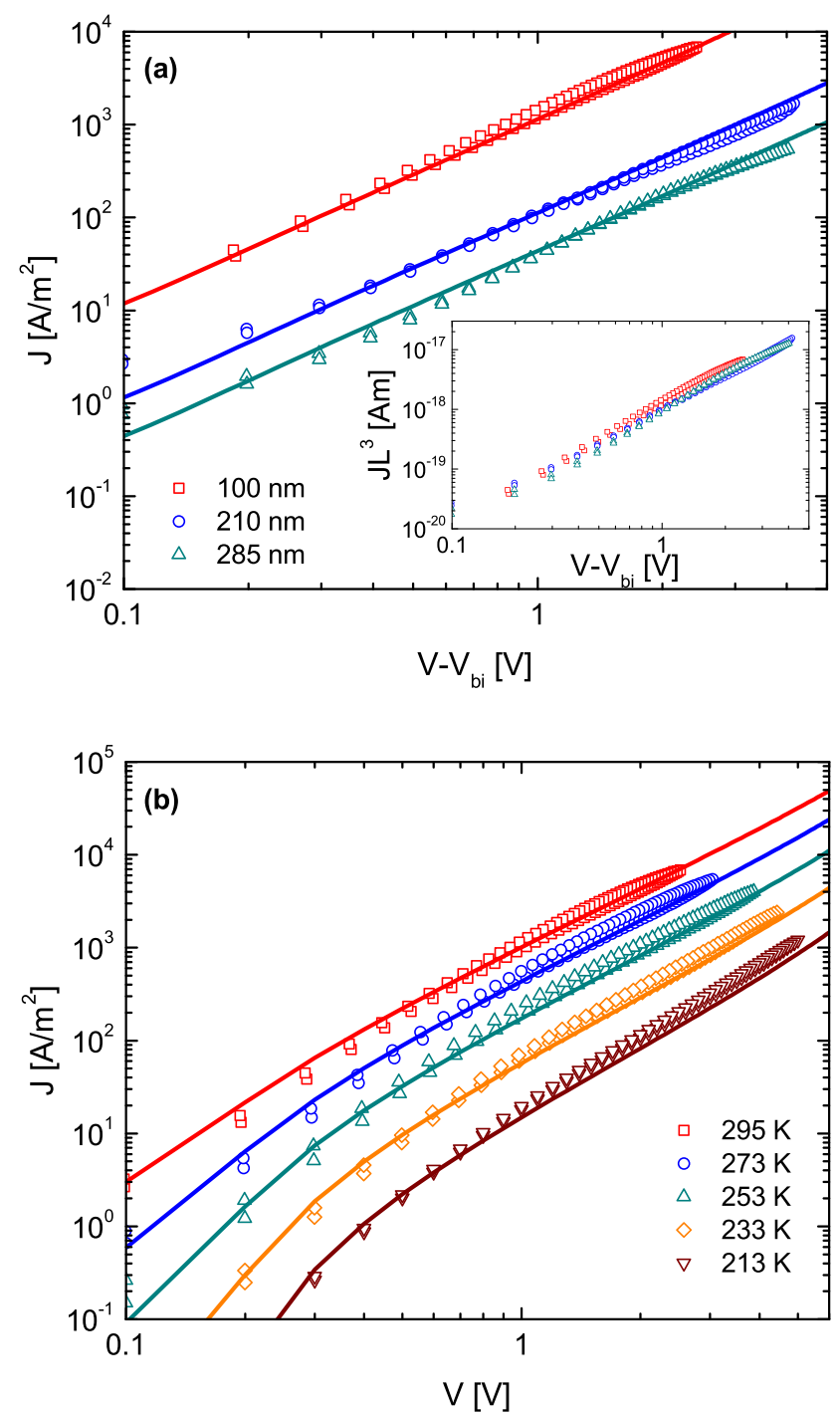

FIG. 2. Experimental (symbols) and simulated (lines) current densityvoltage characteristics of $\alpha$-NPD hole-only devices, for different thicknesses at room temperature (a) corrected for a small built-in voltage $V_{\text {bi }}$ of $0.2 \mathrm{~V}$, and for different temperatures for the $100 \mathrm{~nm}$ device (b). The inset in (a) shows the current density multiplied by $L^{3}$. 


$$
\mu_{0}(T)=\mu_{\infty} c_{1} \exp \left[-c_{2}\left(\frac{\sigma}{k T}\right)^{2}\right]
$$

with $c_{1}=1.8 \times 10^{-8}, c_{2}=0.42$, which are given by the $\mathrm{EDGM},{ }^{22}$ and are similar to other models, ${ }^{23} k$ the Boltzmann constant, and $T$ the temperature. With increasing energetic disorder $(\sigma)$, the temperature, density, and field dependence of the mobility increases. The lattice constant $a$ mainly affects the field dependence of the mobility.

As observed from Fig. 2, the experimental data are consistently described by $1 \mathrm{D}$ drift-diffusion simulations ${ }^{24}$ using the EDGM with a single set of parameters, indicating that the currents are limited by space charge and not by charge injection. The current is calculated from a numerical solution of the drift-diffusion current-flow equation and the Poisson equation, yielding the carrier density, potential, and mobility as a function of position in the device. ${ }^{24}$ The energetic disor$\operatorname{der} \sigma$ is found to be $0.09 \mathrm{eV}$, which is slightly narrower than previously obtained. ${ }^{5}$ The lattice constant $a$ was found to be $0.9 \mathrm{~nm}$, which is consistent with the experimental density of $\alpha$-NPD molecules. ${ }^{13}$ This is an important result considering that the molecular density is a measurable quantity, which would be a step toward a parameter-free description of charge transport in small-molecular semiconductors. A mobility prefactor $\mu_{\infty}$ of $2500 \mathrm{~m}^{2} / \mathrm{Vs}$ was used to described the experimental data, which equates to a room temperature $(T=295 \mathrm{~K})$ mobility of $2.33 \times 10^{-8} \mathrm{~m}^{2} / \mathrm{Vs}$ at vanishing carrier density and electric field. This number is consistent with the time-of-flight mobility for thick films, which was found to be in the range of $2-3 \times 10^{-8} \mathrm{~m}^{2} / \mathrm{Vs}^{25,26}$ In our experiments, however, this mobility was found for film thicknesses down to $100 \mathrm{~nm}$, which are more device relevant. This also shows that the hole mobility in $\alpha$-NPD is not thickness dependent, in contrast to earlier reports. ${ }^{19,20}$ The inset in Fig. 2(a) confirms that the current density indeed scales with layer thickness according to Eq. (1), indicating a spacecharge-limited current.

The value for the energetic disorder of $0.09 \mathrm{eV}$ is lower than earlier reported values of $0.10 \mathrm{eV}$ (Ref. 5) and $0.14 \mathrm{eV}$ (Ref. 13) as obtained from modeling of hole-only devices. While the value of $0.14 \mathrm{eV}$ (Ref. 13) has been attributed to an underestimation of the injection barrier in the modeling, ${ }^{5}$ our results show that the experimental data in Ref. 5 might still be influenced by a charge-injection barrier, resulting in a slight overestimation of the energetic disorder. This would also explain why the calculated mobility in Ref. 5 was an order of magnitude lower than time-of-flight mobilities and our present results.

Having characterized the hole transport, we will now focus on the electron transport. The molecule $\alpha$-NPD is usually referred to as a hole transport molecule, but quantum chemical calculations of the hopping rates ${ }^{27}$ and time-offlight experiments ${ }^{28,29}$ show that the electron mobility is as high or slightly higher than the hole mobility in $\alpha$-NPD.

To investigate the electron transport in $\alpha$-NPD thin films, electron-only devices were fabricated, in which the $\alpha$-NPD layer was sandwiched between an Al bottom electrode and a Ba/Al electron-injecting top electrode. The measured $J-V$ characteristics for different $\alpha$-NPD layer thicknesses are displayed in Fig. 3. It is immediately evident that the electron current is orders of magnitude lower than the hole current. Furthermore, the currents depend more steeply on voltage and have larger thickness dependence. These are fingerprints of a trap-limited electron current. ${ }^{30}$ The presence of electron trapping is confirmed by simulating the thickness-dependent $J$-V characteristics [Fig. 3(a)] with the incorporation of electron traps. The electron currents are well described by the simulations using Gaussianly distributed traps with a trap density of $1.3 \times 10^{24} \mathrm{~m}^{-3}$, a depth of $0.67 \mathrm{eV}$ below the center of the LUMO, and a distribution width of $0.2 \mathrm{eV}$.

In the simulations, the mobility of free electrons was assumed to be equal to the hole mobility, with the same disorder and lattice constant, which is justified by the similar electron mobility as measured by time of flight. ${ }^{28,29}$ In timeof-flight measurements, the transient mobility is hardly affected by the presence of traps deeper than $\sim 0.3 \mathrm{eV} .^{12}$ In electron-only devices, however, the current is heavily reduced: the calculated carrier densities at $10 \mathrm{~V}$ range from $2.3 \times 10^{23} \mathrm{~m}^{-3}$ to $5.5 \times 10^{23} \mathrm{~m}^{-3}$ for the $146 \mathrm{~nm}$ to the
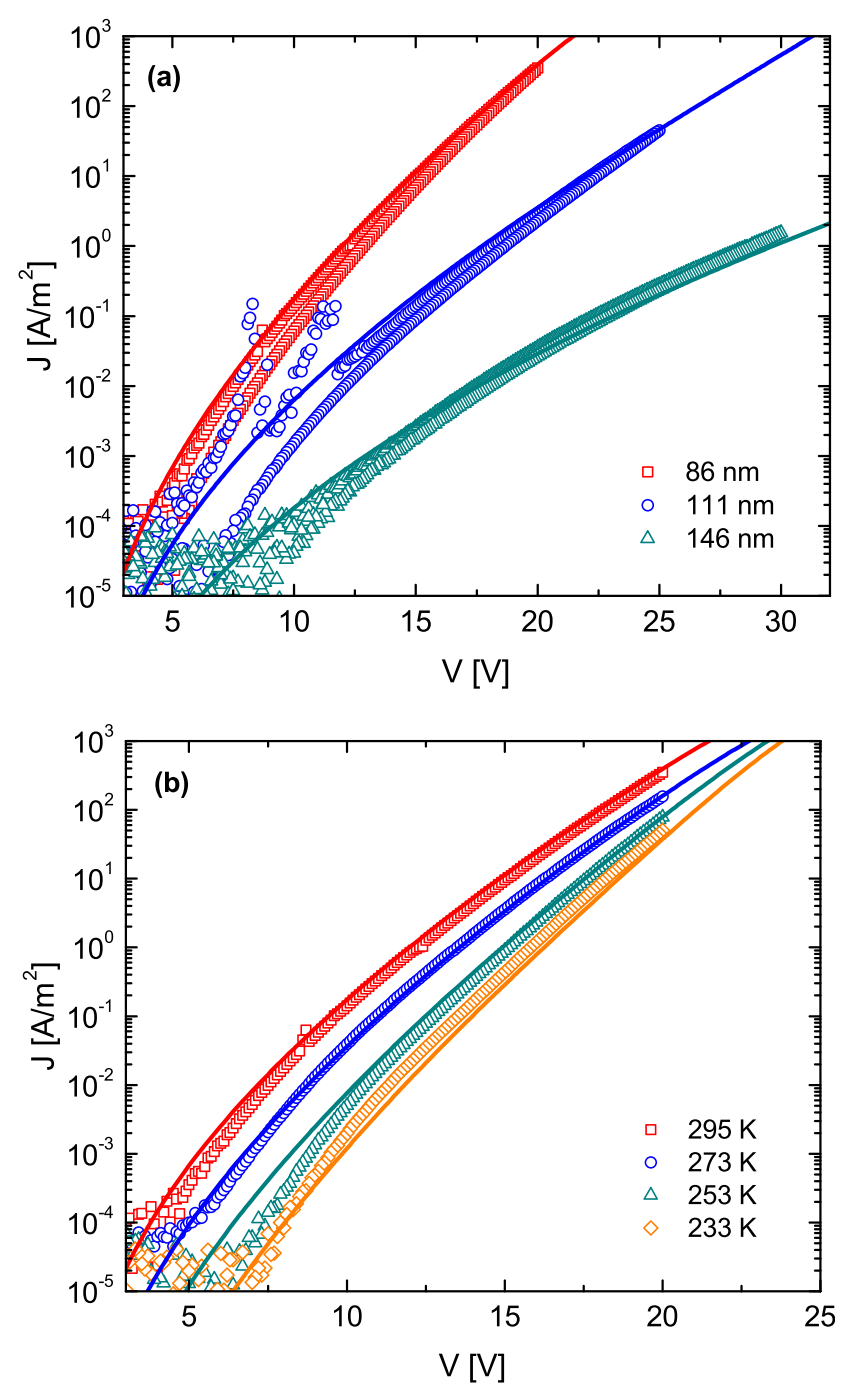

FIG. 3. Experimental (symbols) and simulated (lines) current densityvoltage characteristics of $\alpha$-NPD electron-only devices, for different thicknesses at room temperature (a), and for different temperatures for the $86 \mathrm{~nm}$ device (b). 
$86 \mathrm{~nm}$ device, indicating that almost all carriers are immobilized in the electron traps.

Even though in many cases it is not possible to clearly determine the exact trap distribution (Gaussian vs exponential) from trap-limited currents, ${ }^{31}$ the electron currents cannot be described without introducing a high concentration of electron traps. A simple reduction of the mobility or the incorporation of an injection barrier cannot reproduce the experimental data.

The temperature-dependent electron current in Fig. 3(b) can be simulated by adjusting the effective trap depth $E_{t, e f f}$ for different temperatures according to ${ }^{31}$

$$
E_{t, e f f}=E_{t, a b s}-\frac{\sigma_{L U M O}^{2}}{2 k T},
$$

where $E_{t, a b s}$ is the absolute trap depth with respect to the center of the LUMO and $\sigma_{L U M O}$ is the width of the Gaussian distribution of LUMO states. The effective trap depth arises from the energetic disorder of LUMO states and is defined as the depth of the trap with respect to the effective conduction-band edge, situated at a temperaturedependent value of $\sigma^{2} / 2 k T$ below the center of the LUMO.$^{30,31}$ By fitting the temperature dependence of the electron current with a temperature-dependent $E_{t, \text { eff }}$ according to Eq. (3), a good description could be obtained with $E_{t, a b s}=0.67 \mathrm{eV}$ and $\sigma_{L U M O}=0.105 \mathrm{eV}$, in good agreement with the value of $0.09 \mathrm{eV}$ found from modeling the hole transport with the EDGM.

Trap-limited electron currents are frequently found in conjugated polymers, which have been attributed to the presence of extrinsic impurities, ${ }^{7}$ with water-oxygen complexes being a likely candidate. For organic small molecules however, there is no clear consensus on the electron transport and trapping behavior. In the aluminum chelates $\mathrm{Alq}_{3}$ and BAlq, electron transport was found to be limited by trapping, ${ }^{14,15}$ albeit to a far lesser extent than in $\alpha$-NPD. The existence of such pronounced trapping effects is remarkable, since, unlike solution-processed conjugated polymer films, the layers are deposited in a high vacuum. In addition, organic small molecules are monodisperse, do not feature end groups, and are easier to purify. Nevertheless, the calculated trap concentration for $\alpha$-NPD is in the same range or even higher than the trap densities in most conjugated polymers. ${ }^{7}$ Considering the amorphous nature of $\alpha$-NPD films, ${ }^{16,17}$ it is not likely that aggregates or grain boundaries are responsible for the electron trapping, also considering that the hole transport is trap free. In $a b$ initio simulations, the energetic distribution for electron transport was found to be even lower than for hole transport, ${ }^{17}$ which would imply that the electron traps found here are extrinsic defects and not related to structural disorder. As trapping sites not only limit the charge transport but also act as nonradiative recombination centers, it is highly important to find the origin of these defects. While often disregarded in OLED research, our study shows that electron trapping in evaporated small molecules plays an important role.

In summary, trap-free hole transport and trap-limited electron transport were found in the archetypical small molecule $\alpha$-NPD. The hole mobility of $2.33 \times 10^{-8} \mathrm{~m}^{2} / \mathrm{Vs}$ extracted from space-charge-limited current measurements was found to be in close agreement with reported time-offlight mobilities. Applying the EDGM to the experimental hole currents resulted in a relatively narrow energetic disorder of $0.09 \mathrm{eV}$ and a modeled lattice constant of $0.9 \mathrm{~nm}$, close to the average intermolecular distance. Remarkably, the electron currents were found to be orders of magnitude lower than the hole currents in single-carrier devices, which was assigned to the presence of a high concentration of electron traps of $1.3 \times 10^{24} \mathrm{~m}^{-3}$. The similarity of such a strongly trap-limited electron current in small molecules and conjugated polymers is remarkable, since the molecules are vacuum deposited and are monodisperse, whereas conjugated polymers are polydisperse and processed from solution.

This project has received funding from the European Union Horizon 2020 research and innovation programme under Grant Agreement No. 646176 (EXTMOS).

${ }^{1}$ C. W. Tang and S. A. Van Slyke, Appl. Phys. Lett. 51, 913 (1987).

${ }^{2}$ C. W. Tang, Appl. Phys. Lett. 48, 183 (1986).

${ }^{3}$ H. Bässler, Phys. Status Solidi B 175, 15 (1993).

${ }^{4}$ F. May, B. Baumeier, C. Lennartz, and D. Andrienko, Phys. Rev. Lett. 109, 136401 (2012).

${ }^{5}$ A. Massé, P. Friederich, F. Symalla, F. Liu, R. Nitsche, R. Coehoorn, W. Wenzel, and P. A. Bobbert, Phys. Rev. B 93, 195209 (2016).

${ }^{6}$ M. Mesta, M. Carvelli, R. J. de Vries, H. van Eersel, J. J. M. van der Holst, M. Schober, M. Furno, B. Lüssem, K. Leo, P. Loebl, R. Coehoorn, and P. A. Bobbert, Nat. Mater. 12, 652 (2013).

${ }^{7}$ H. T. Nicolai, M. Kuik, G. A. H. Wetzelaer, B. de Boer, C. Campbell, C. Risko, J. L. Brédas, and P. W. M. Blom, Nat. Mater. 11, 882 (2012).

${ }^{8}$ G. A. H. Wetzelaer, M. Kuik, H. T. Nicolai, and P. W. M. Blom, Phys. Rev. B 83, 165204 (2011).

${ }^{9}$ R. G. Kepler, Phys. Rev. 119, 1226 (1960).

${ }^{10}$ P. W. M. Blom and M. C. J. M. Vissenberg, Phys. Rev. Lett. 80, 3819 (1998).

${ }^{11}$ N. I. Craciun, J. J. Brondijk, and P. W. M. Blom, Phys. Rev. B 77, 035206 (2008).

${ }^{12}$ C. Li, L. Duan, H. Li, and Y. Qiu, J. Phys. Chem. C 118, 10651 (2014).

${ }^{13}$ S. L. M. van Mensfoort, V. Shabro, R. J. de Vries, R. A. J. Janssen, and R. Coehoorn, J. Appl. Phys. 107, 113710 (2010).

${ }^{14}$ W. Brütting, S. Berleb, and A. G. Mückl, Org. Electron. 2, 1 (2001).

${ }^{15}$ S. L. M. van Mensfoort, R. J. de Vries, V. Shabro, H. P. Loebl, R. A. J. Janssen, and R. Coehoorn, Org. Electron. 11, 1408 (2010).

${ }^{16}$ D. S. Qin, D. H. Yan, and L. X. Wang, Appl. Phys. Lett. 77, 3113 (2000).

${ }^{17}$ P. Friederich, F. Symalla, V. Meded, T. Neumann, and W. Wenzel, J. Chem. Theory Comput. 10, 3720 (2014).

${ }^{18}$ N. F. Mott and R. W. Gurney, Electronic Processes in Ionic Crystals (Oxford University Press, London, 1940).

${ }^{19}$ T. Matsushima, Y. Kinoshita, and H. Murata, Appl. Phys. Lett. 91, 253504 (2007).

${ }^{20}$ T. Y. Chu and O. K. Song, Appl. Phys. Lett. 90, 203512 (2007).

${ }^{21}$ M. Kröger, S. Hamwi, J. Meyer, T. Riedl, W. Kowalsky, and A. Kahn, Org. Electron. 10, 932 (2009).

${ }^{22}$ W. F. Pasveer, J. Cottaar, C. Tanase, R. Coehoorn, P. A. Bobbert, P. W. M. Blom, D. M. de Leeuw, and M. A. J. Michels, Phys. Rev. Lett. 94, 206601 (2005).

${ }^{23}$ L. Li, N. Lu, and M. Liu, EPL 106, 17005 (2014).

${ }^{24}$ L. J. A. Koster, E. C. P. Smits, V. D. Mihailetchi, and P. W. M. Blom, Phys. Rev. B 72, 085205 (2005).

${ }^{25}$ B. Chen, C.-S. Lee, S.-T. Lee, P. Webb, Y.-C. Chan, W. Gambling, H. Tian, and W. Zhu, Jpn. J. Appl. Phys. 39, 1190 (2000).

${ }^{26}$ S. C. Tse, S. W. Tsang, and S. K. So, J. Appl. Phys. 100, 063708 (2006).

${ }^{27}$ B. C. Lin, C. P. Cheng, and Z. P. M. Lao, J. Phys. Chem. A 107, 5241 (2003). 
${ }^{28}$ S. C. Tse, K. C. Kwok, and S. K. So, Appl. Phys. Lett. 89, 262102 (2006). ${ }^{29}$ S.-W. Liu, C.-C. Lee, W.-C. Su, C.-H. Yuan, C.-F. Lin, K.-T. Chen, Y.-S. Shu, Y.-Z. Li, T.-H. Su, B.-Y. Huang, W.-C. Chang, and Y.-H. Liu, Sci. Rep. 5, 10384 (2015).
${ }^{30}$ M. M. Mandoc, B. de Boer, G. Paasch, and P. W. M. Blom, Phys. Rev. B 75, 193202 (2007).

${ }^{31}$ H. T. Nicolai, M. M. Mandoc, and P. W. M. Blom, Phys. Rev. B 83, 195204 (2011). 